\title{
La danza en las políticas públicas: Un mundo por construir
}

doi: 10.33264/rpa.201901-06

Helena Hidalgo G

Escuela de Danza y Coreografía

Facultad de Artes de la Comunicación, UNIACC

\section{Resumen}

Danza y políticas públicas son dos conceptos que parecen distantes, sin embargo, en Chile a partir de la creación de la política de fomento generada para su desarrollo, por el entonces Consejo Nacional de la Cultura y las Artes, cubriendo el período 2010-2015, dejan de serlo y, resulta fundamental revisar cuál es la propuesta de esta política, cuál su diseño de implementación, presupuesto, resultados esperados y obtenidos y, por cierto, cuál su proceso de continuidad.

El presente artículo constituye una mirada inicial a esta propuesta de política pública, en pos de las posibilidades de participación de las instituciones de educación superior, que cuentan con una oferta educativa profesional para la danza.

Palabras clave: danza, políticas públicas.

\section{Abstract}

Dance and public policies are two concepts that seem distant, however, in Chile from the creation of the promotion policy generated for its development, by the then National Council of Culture and Arts, covering the period 2010-2015, they cease to be and, it is essential to review what is the proposal of this policy, what its implementation design, budget, expected and obtained results and, by the way, what its continuity process.

This article constitutes an initial look at this public policy proposal, in pursuit of the possibilities of participation of higher education institutions, which have a professional educational offer for dance.

Keywords: dance, public policy. 


\section{Introducción}

El presente artículo realiza un análisis de lo que es la política de fomento a la danza para el período 2010-2015, formulada por el Consejo de la Cultura y las Artes.

Resulta interesante analizar esta propuesta, pues es la primera vez que el Estado tras ocuparse de construir un diagnóstico de la situación de la danza nacional, encuentra pertinente y necesario establecer una política de fomento para esta rama del arte de manera diferenciada, teniendo en cuenta que las artes escénicas o de la representación suelen ser englobadas en una sola, donde la danza es casi imperceptible tras el teatro y la actuación, tal como lo demuestra la designación del Premio nacional de Artes de la representación y audiovisuales, donde la premiación a la danza ha sido exigua desde su creación y, nunca considerada mientras existió en la categoría global de Premio Nacional de Artes, razón por la cual una política específica es significativa y notable.

Podría resultar esperable la existencia de la misma, la generación de una institucionalidad pública cuyo objetivo específico es la cultura y el arte, como es el caso del Consejo de la Cultura que surge a mediados del año 2003, durante el gobierno del Presidente Ricardo Lagos, el que se transforma en Ministerio en febrero de 2018, durante el segundo gobierno de la Presidente Michelle Bachelet, convierte en un imperativo el surgimiento de la política pública referida de manera diferenciada y específica a cada una de las materias que constituyen el objetivo del Consejo, no obstante no deja de ser destacable la primera vez para una política pública en danza. No es dable ignorar tamaño avance.

Teniendo en cuenta que las artes son variadas y más de una, además de una política pública global que permita y facilite el desarrollo simultáneo de todas ellas, la generación de políticas específicas por arte, es un avance que va más allá y constituye un logro mayor que merece una lectura minuciosa. Por otro lado, pocas veces las políticas públicas son declaradas por el Estado con la claridad con que lo ha sido la política en danza, la que no solo contiene un marco histórico referencial, sino también un diagnóstico, una agrupación temática de las dificultades y, una propuesta de desarrollo para superarlas.

Este artículo es un análisis inicial, sin grandes pretensiones, de carácter exploratorio y de un objetivo simple: Analizar la propuesta de fomento a la danza para los años 2010-2015 y, visualizar cuáles son las posibilidades de participación de las instituciones de educación superior que cuentan con una oferta formativa en danza. 
Tratándose de un artículo, no cuenta con capítulos que lo organicen, pero sí con subtítulos que guían los tópicos a los que alude, en términos de qué se plantea cómo política, cuál es la información con que se cuenta respecto de su desarrollo, los diferentes aspectos que se visualizan de ella $y$, en segundo lugar, cuáles son las posibilidades de participar desde las instituciones de educación superior que consideran la danza como parte de sus opciones educativas.

\section{Objetivo general}

Revisar la propuesta de fomento a la danza para los años 2010-2015, realizada por el Consejo Nacional de la Cultura y las Artes de Chile, en pos de encontrar las opciones de participación de las instituciones de educación superior que cuentan con dicha formación.

\section{Metodología}

El presente artículo se inscribe fundamentalmente en la categoría de estudio exploratorio, en la medida que plantea “... examinar un tema o problema poco estudiado o que no ha sido abordado antes. Es decir, cuando la revisión de la literatura reveló que únicamente hay guías no investigadas e ideas vagamente relacionadas con el problema de estudio." (Hernández, 1997, p.70).

Dicho de otra manera, artículo exploratorio en su enfoque en tanto no mide ni evalúa, quizás en algunos aspectos se acerque más a lo explicativo, en tanto describe eventos o causas que pudieran constituir la razón o parte de ella para una determinada situación. Es además de exploratorio, un análisis no experimental, pues utiliza un material que ha sido seleccionado de manera no probabilística, lo que permite mayor libertad en la recopilación y análisis de la información, en especial, en lo que concierne a la incorporación de nuevas preguntas. En resumen, un trabajo que puede modificar y/o ampliar el universo de preguntas a responder, toda vez que trata de una materia que inicia su desarrollo en Chile.

\section{Danza y políticas y públicas, el inicio del recorrido}

Para Lasswell ${ }^{21}$, iniciador de la disciplina conocida como políticas públicas, ésta no se ocupa de temas puntuales sino de problemas de relevancia para el ser humano en sociedad, los que dicen relación con su dignidad humana, situando a las políticas

\footnotetext{
${ }^{21}$ Harold Lasswell, (13 de febrero de 1902 a 18 de diciembre de1978) experto en comunicaciones y analista de políticas, reconocido como el iniciador de la disciplina de las políticas públicas.
} 
públicas en un espacio de democracia, en tanto no buscarían el control sino la mejora de una situación de vida que involucra a muchos, donde las propuestas de salida al problema requieren creatividad y participación, pues no es posible superar el problema con un simple barniz que optimiza lo que ya existe, se requiere algo más de esfuerzo dado que no hay más opción que transformar, que modificar la realidad si el objetivo es superar, aunque sea en parte, el problema declarado.

Las políticas públicas son entonces un área de estudio que requiere libertad de pensamiento y expresión pues, entre otras cosas, resulta evidente que la posición desde donde se realiza el análisis del problema y cómo enfrentarlo no es neutra, problema y solución cuentan siempre con una expresión valórica, máxime cuando hablamos de arte, que muestra el proyecto de sociedad del cual se participa y se busca apoyar, en tanto se considera importante o no la existencia y acceso de un algo para toda la ciudadanía o, se le considera irrelevante para el desarrollo social, lo que en este caso resulta primordial tener en cuenta dada la habitual concepción del arte como un "excedente", esto es, un elemento suntuario que no forma parte de las necesidades fundamentales del desarrollo humano y, por ende, un bien de consumo al cual se accede de contar con los recursos que lo permitan.

El documento "Política de fomento de la danza 2010-2015" elaborado y publicado por el que fuera el Consejo de la Cultura de Chile, constituye un interesante e importante análisis de la situación de la danza en Chile toda vez que corresponde a un análisis realizado por el Estado, y da cuenta de cuál ha sido su camino inicial en el país hasta lo que es su situación actual de este arte o, al menos hasta el año 2015, fecha tope del documento. En él se declara sin tapujos por parte del Estado, uno a uno los problemas o falencias (que no es lo mismo, pero al caso, resulta igual) para el desarrollo de la disciplina de la danza y, presenta una propuesta para cada uno de los aspectos deficitarios que se identifican.

Destacar que el documento se plantea como una política de fomento, esto es, como un instrumento de promoción e impulso para la danza nacional, sin apellidos, simplemente danza, la considerada como arte: clásica, moderna, contemporánea, de espectáculo de vanguardia, no hay distingo, se trata de buscar el desarrollo de un arte escénica en sus múltiples estilos y expresiones. Se la considera una política “... conjunta entre el Estado y la sociedad civil.", algo así como bailemos juntos. El aludido documento se plantea un horizonte de cinco años (2010-2015) para su desarrollo, y al momento de lanzarla, se da cuenta de lo que fuera su proceso de construcción, el que se inicia el año 2006, después de un largo y participativo trabajo realizado con representantes del mundo de la danza, aspecto también destacable: una revisión colectiva de la situación entre Estado y sociedad civil es el mejor de los 
mundos para ver y conocer la situación sobre la cual se desea intervenir, pues de ello deriva una propuesta donde la sociedad civil junto con sentirse partícipe, tiene espacios reales para incorporarse al desarrollo de la política en calidad de constructor y beneficiaro.

Entonces, cuando un Estado decide dedicarle tanto tiempo y aunque no diga cuánto, también recursos, a la elaboración de una política en un área del desarrollo cultural humano como es la danza y, además lo plantea como una propuesta colectiva en tanto así se construye y así se espera implementar, realizar un análisis para esta política desde el mundo social resulta primordial, ineludible. Quizás habría que destacar también que la incorporación del arte al mundo de las políticas públicas en sociedades como las nuestras, donde cuesta pensar que éste sea una necesidad y no un excedente, es para aplaudir de pie.

La política en comento establece cinco lineamientos estratégicos, desde la perspectiva del análisis podría decirse que establece cinco problemas fundamentales: creación artística; promoción y comercialización; participación, acceso y formación de audiencia; patrimonio cultural y, por último, institucionalidad. Para cada una de éstas áreas, además del análisis que permite su identificación como problema, también se establecen objetivos y una propuesta en pos de la superación de los mismos.

Con posterioridad a este documento, surge la renovación de la política pública global en cultura a cargo del Consejo Nacional de la Cultura y las Artes, la que cubre un espacio temporal entre los año 2017-2022, una vez más quinquenal y la última declaración de política pública del Consejo, pues con posterioridad y a partir del año 2018 vendrá la transformación a Ministerio de las Culturas y las artes.

Este documento de política pública 2017-2022, cuenta con una mirada amplia en pos del desarrollo del concepto de "ciudadanía cultural” (el que podría entenderse como la apropiación de la cultura, el arte y el patrimonio por parte del o la ciudadana, no importando su lugar de residencia y si es creador/a o no, pero ese es un tema para otro artículo), pero previo a él están "Chile quiere más cultura. Definiciones de política cultural 2005-2010" y “Política Cultural 2011-2016". Podría decirse, que la política pública en danza no surge de la nada, existe toda una propuesta, una línea base robusta respecto de cómo el Estado ve y considera debe asumir el desarrollo de la política pública de las artes en Chile. Teniendo en cuenta que todos ellos emanan de la primera institucionalidad pública en cultura y arte, son documentos que constituyen ejes fundacionales, el referente a tener siempre en cuenta al momento de generar las evaluaciones. 
Gran trabajo que no termina en lo expuesto pues, al revisar la página web del ayer Consejo de la Cultura y hoy Ministerio, en la sección que corresponde al Departamento de estudios se puede encontrar toda un área dedicada al desarrollo de las políticas según tipo de expresión artística, agrupadas todas en una "Colección de políticas sectoriales de cultura". También existe toda una línea dedicada al análisis estadístico, encuestas, tesis que han sido financiadas por tratar de arte, consultorías de variados temas de importancia efectiva para el desarrollo de la cultura y el arte, pero no se registran un balance de las políticas específicas como la de danza, no resultando factible conocer presupuesto, programas, logros en cada una de las áreas establecidas como estratégicas. Ampliando la búsqueda de información, a simple revisión de la web de la Dirección de Presupuesto, DIPRES, tampoco aparece algún tipo de estudio que diga relación con la inversión en la política de Danza. De seguro, algo de presupuesto del Estado se ha utilizado en la implementación de esta declaración de política pública en danza como en la de otras artes, sin embargo, no resulta posible encontrar información de manera sencilla donde se hubiere registrado y sistematizando ese tipo de información, al menos este año 2019, momento de elaboración del presente artículo.

Por ello, en lo que respecta a la política pública específica para danza, no resulta posible conocer sus resultados tanto en relación a su inversión como en lo concerniente a sus avances en cuanto a logros, aunque no está demás decir que el documento que contiene la política en danza, en ninguna de sus partes señala cuál es el presupuesto destinado para implementar, fomentar o desarrollar las propuestas declaradas, como tampoco se enuncia un espacio de balance o evaluación respecto del estado de avance, dificultades y logros que pudieran producirse durante el umbral de cinco años que cubre. No es condición de una política hacerlo, pues un aspecto es la declaración de la política pública y otra, complementaria, consecutiva pero diferente, su diseño, peldaño posterior sobre el cual se construye la implementación o la operativización misma de la política pública, espacios donde los procesos de evaluación y definición de presupuesto sí son condición. En pocas palabras, la política de danza es una especie de mesanina desde donde surge una pequeña escalera que por el momento no podemos utilizar para subir o bajar, la revisión y análisis de lo realizado será un avance parcial hasta contar con toda la información que permitirá un análisis cada vez más completo en la medida que el tiempo avance, el arte tiene quizás tiempos distintos en estos aspectos, resulta evidente que dada su especialidad, no necesariamente es lo mismo que una política pública en otra materia.

Una política pública podría decirse constituye un curso de acción, donde se encuentra la ruta diseñada y la efectivamente seguida, en otras palabras, es lo que el gobierno dice y quiere hacer, marcando en dos columnas diferentes pero paralelas, el resultado 
o las pautas del resultado esperado de, lo que realmente hace y logra, la tercera columna no es el resultado necesariamente, sino el diferencial de lo que resta por hacer en términos de continuidad o cambio de ruta parcial o total. El documento en comento es una política pública declarada, no cabe duda, y como ya se dijo, en sociedades como la nuestra, con importantes problemas a resolver para que seres humanos y naturaleza puedan contar con una vida digna, justa para todos y todas, en igualdad de oportunidades y condiciones, lo que debiera incluir la cultura y la danza como parte de ella, no es una preocupación de Estado tradicional en Chile, y es justamente por ello que el análisis desde la perspectiva de las políticas públicas es a la hora de una apropiada decisión una tarea ineludible para los actores involucrados en ella.

Evidentemente las políticas públicas constituyen el trabajo del Estado frente a las diferentes materias de las cuales le corresponde hacerse cargo, y la danza en tanto constituyente del desarrollo cultural y social de nuestro país, es parte de sus responsabilidades, no obstante resulta también pensable ¿cuál es el rol y la participación que le corresponde a la sociedad civil, al mundo privado y al propio gremio de la danza dentro del mundo de las políticas públicas, considerando que somos parte del Estado en tanto ciudadanos y ciudadanas?

Lahera $^{22}$, una eminencia en las políticas públicas chilenas, conocedor de nuestra realidad, decía que una política pública “... corresponde a cursos de acción y flujos de información relacionados con un objetivo público definido en forma democrática; los que son desarrollados por el sector público y, frecuentemente, con la participación de la comunidad y el sector privado. Una política pública de calidad incluirá orientaciones o contenidos, instrumentos o mecanismos, definiciones o modificaciones institucionales, y la previsión de sus resultados." (Lahera, 2002, p.16). Esta definición permite saber de qué se ocupa, quiénes participan de la política y, fija el estándar de logro de esta política. Es una definición que cubre el trayecto desde la declaración del objetivo, su diseño (momento en que al menos debe contarse ya con un presupuesto exploratorio), implementación (etapa en que el presupuesto debe estar establecido) y resultados (análisis donde el éxito de la política no necesariamente se debe medir en relación a los recursos destinados).

Entonces, retomando el mentado documento sobre la política pública de danza Chile, la danza ha sido visibilizada como parte de los problemas, tareas, áreas de desarrollo

\footnotetext{
22 Eugenio Lahera Parada (1948-2012). Destacado analista de políticas públicas, abogado de la Universidad de Chile, Ph.D. y Master en Public Affairs de la Universidad de Princeton, fue uno de los primeros doctores en políticas públicas en Chile.
} 
de la política pública nacional. Es un documento que fija un curso de acción pues señala cuáles son las áreas deficitarias sobre las cuáles trabajar, presenta un diagnóstico, un análisis en base a él, da antecedentes, relaciona los diferentes aspectos problemáticos y define cinco espacios sobre los cuales intervenir para avanzar. Ya está dicho que no pone presupuesto, actores, plazos, espacios de evaluación, ello porque la declaración de una política pública no constituye su diseño, ese es el paso que viene a continuación y es ahí donde se abren las grandes alamedas teniendo en cuenta que la ejecución, implementación u operativización, como quiera llamársele, donde se puede ver si todo lo propuesto efectivamente permite un cambio de situación hacia una realidad mejor.

Pero entre la declaración de una política, su diseño y su ejecución, existen cambios de administración en el gobierno que pueden ser drásticos, generando eventuales demoras, cambios de énfasis, reducción de presupuesto. En países como Chile ¿dónde quedan las políticas públicas del gobierno anterior cuando se da un cambio de administración con enfoques ideológicos diferentes?, ¿cómo resolver la continuidad del proyecto?, ¿cómo visualizar los avances cuando la perspectiva valórica se modifica? o, de no existir un cambio de gobierno con grandes contrastes, ¿puede presumirse continuidad de lo que ya venía en proceso, incluso podría implicar aumento de énfasis en algunos aspectos?, eso depende de múltiples variables, en general relacionadas con la situación económica o problemas emergentes tales como catástrofes nacionales. Este aspecto, el de cambio de gobierno, es un problema que dada la experiencia desde los años '9o no ha mostrado mecanismos de solución, pues mientras dichos cambios han respondido a una idea de desarrollo social similar, las políticas públicas se han mantenido o contado con leves variaciones, pero cuando los cambios de administración constituyen una propuesta claramente distinta de desarrollo social, las variaciones en la política pública han sido sustantivas.

Conocer los fundamentos de la política pública es un paso tan necesario como la existencia de los estudios de evaluación que permiten conocer sus resultados, no importando si éstos pudieran ser desalentadores o auspiciosos, si dan cuenta de un momento específico o de toda una época, ello porque son antecedentes que facilitarían una mayor comprensión de los aportes de tal o cual objetivo en pos de los cambios, avances, proyecciones buscadas, el que no existan o, aun existiendo no sean suficientes, no resulta ser un problema solamente, es también la oportunidad para que, por ejemplo, los centros de pensamiento como son las instituciones de educación superior puedan trabajar de manera independiente y/o en conjunto con el Estado, en alguno de los temas que tratan estos cinco ejes en el caso de la política que constituye el eje del presente artículo, ello abrirá la conversación tan necesaria cuando se participa del proceso de formación de profesionales que se incorporarán 
al mercado laboral nacional haciendo parte del desarrollo país, constituyendo el capital social de una nación y, cuyo proyecto de vida cuenta con algunas diferencias, pues se trata de uno que durante décadas ha sido visto como un "excedente", toda vez que la creación es una "actividad recreativa", un "hobby", no un proyecto de desarrollo profesional equivalente a las áreas tradicionales como la ciencia o las matemáticas, vistas como un aporte al desarrollo.

\section{La participación de las instituciones de educación superior en la política pública de la danza en Chile}

Ya en su diagnóstico, la política en danza señala que “... en su dimensión social o educativa y mediante procesos de enseñanza y aprendizaje, la danza produce revelaciones a través de la propia experiencia corporal que resultan imprescindibles para el desarrollo integral del ser humano. En este sentido, aparece como fundamental incorporar la danza en la educación." (C.N.C.A., 2010,p 10). En otras palabras, se visualiza a la danza desde la formación inicial de las personas, no solo al momento del pregrado. Puede resultar evidente para quienes participan o han participado alguna vez del mundo de la danza, pues el oficio de intérprete suele iniciar a temprana edad, y los beneficios de la danza en las denominadas competencias transversales y el desarrollo emocional de las personas se han destacado de manera creciente con los años, no obstante para la generalidad, la danza no suele formar parte de los procesos formativos de las personas.

Más adelante se dedican unos párrafos expresos a lo que se considera el espacio específico de danza y educación, para el cual se establecen la educación formal, no formal, e informal. Para la primera se señalan la educación general, la educación superior y las escuelas artísticas. Para la segunda toda una oferta que se la declara "aprendizaje intencional" y que es de variado carácter pero siempre estructurado en calidad de talleres o similares y, para la tercera y última se habla de una educación "no estructurada", de carácter social referida a las expresiones religiosas y folklóricas. Resulta entonces, que la danza se encuentra inserta en todos los niveles educativos que se reconocen en el país, situación que no se consigue de un día para otro, es decir la danza forma parte del desarrollo social y cultural del país, aunque no siempre se hubiere visto o reconocido su existencia.

Continuando con el diagnóstico, el documento dedica un espacio a lo que se denomina "Ámbito de formación" donde se incluye la formación profesional en danza, se marca el inicio de este proceso en los años 40 al alero de la Universidad de Chile y su facultad de artes, espacio que siempre ha existido y que se mantuvo después del golpe de Estado y, al que se fueron sumando con el tiempo otras ofertas, relevantes todas, pero no fue hasta el año 1985 en que se agrega la formación 
pedagógica a través de Arcis, la que a pesar de haber desaparecido junto con dicha universidad, continúa a través de otras instituciones de educación superior que la han incorporado a su disponibilidad educativa.

El análisis de la política culmina con el acápite que denomina "Líneas estratégicas" donde “... se presentan los lineamientos necesarios para el desarrollo de la danza como materia de política pública en base a las cinco características principales: Creación artística; Promoción y comercialización; Participación, acceso y formación de audiencias; Patrimonio cultural; e. Institucionalidad". (C.N.C.A., 2010,p 17). Este es el momento en que se identifican las condiciones de precariedad en que la danza ha subsistido durante décadas no importando la línea estratégica sobre la cual se hable, el diagnóstico es detallado y no se escatima espacio en su desarrollo. De este análisis de líneas estratégicas, surgen los objetivos de la política y, es aquí donde aparece todo un mundo por construir, porque resulta innegable que no importando las dificultades “... ahí está la danza de las políticas públicas”23.

Para el lineamiento Creación artística, ya en la sección donde se concreta la propuesta de política misma, se señala como problema central un "Entorno creativo reducido y en condiciones precarias con escasas posibilidades de proyección y sustentabilidad" (C.N.C.A., 2010,p 43), haciendo parte de él 6 problemas específicos, donde varios de ellos podrían reclamar la presencia de las instituciones de educación superior, no obstante hay uno que lo hace de manera más directa y es el referido a la "Oferta formativa muy reducida, escaso número de instituciones de formación profesional a nivel nacional, falta de especialización y ausencia de criterios de calidad y acreditación" (C.N.C.A., 2010,p 43). El diagnóstico de la política pública da cuenta que la formación de pregrado en educación superior en danza existe solo en dos regiones del país, siendo una de ellas la Región Metropolitana, que a la vez es la que cuenta con más opciones, quedando el resto del país desprovisto de la posibilidad de estudiar danza en cualquiera de sus opciones (intérprete o pedagogía), pero lo que resulta aún más complejo, es la ausencia de formación de post grado en danza, no existiendo magíster o doctorado y, con una exigua oferta en diplomado.

¿Cómo construir una carrera profesional en danza si las instituciones de educación superior no cuentan con una oferta educativa de post grado?, ¿cómo avanzar en el mercado laboral siendo docente, intérprete, coreógrafo, director de compañía si no hay diplomados, magíster, doctorado en las diferentes áreas que comprende un arte

\footnotetext{
23 Interesante frase de la que me permito "apropiarme", y que fuera parte de los comentarios que hizo Inés Recuero al presente artículo al momento de su escritura, profesional con años de experiencia en la gestión pública.
} 
escénica como la danza?, ¿quién más se hace cargo de ello si no son las instituciones de educación superior?

En relación a este aspecto, la política establece un espacio que se denomina propuesta de implementación, donde una de ellas es "Realizar un estudio diagnóstico que permita elaborar una estrategia de acercamiento y vinculación con entidades públicas y privadas que impartan estudios especializados de danza, con el fin de potenciar la descentralización de la disciplina". (C.N.C.A., 2010,p 44). En otras palabras, la disposición está, la claridad de por dónde empezar también, es cuestión ahora que las instituciones de educación superior tomen la palabra e inicien el proceso, donde no solo se trata de completar la formación superior para especialización, sino también la extensión de estas opciones como las de pre-grado a regiones, donde la danza no figura como posibilidad de formación, sin dejar de lado las condiciones contractuales del cuerpo docente que se hará cargo de esta labor.

En lo que concierne al lineamiento Participación, acceso y formación de audiencias, éste cuenta con dos problemas centrales identificados, uno de ellos es "Insuficiente inserción de la danza en la educación formal." (C.N.C.A., 2010,p 47), para el cual los objetivos específicos en pos de buscar un cambio contienen las siguientes opciones: "Proponer programas específicos para el aprendizaje de la danza en el sistema educacional, desde la educación parvularia hasta la enseñanza media (medida en Institucionalidad); Promover la incorporación de profesores especialistas en danza, para la adecuada enseñanza de la disciplina en los talleres de formación escolar (medida en Institucionalidad); Diseñar estrategias de formación de audiencias en las escuelas, liceos y educación superior, para. El conocimiento y apreciación de la disciplina." (C.N.C.A., 2010,p 47).

En relación a ello, la propuesta de implementación que se desarrolla dice que "Diseñar e implementar un programa de formación de público que considere experiencias existentes y responda a la sensibilización y valoración del espectador frente a todos los ámbitos en que esta disciplina se desarrolla. Dicho programa deberá considerar su ejecución en etapas y sectorizado a estudiantes del sistema educacional y público en general." (C.N.C.A., 2010,p 47). Pero incluso, aunque este espacio de desarrollo toca a la educación formal de pre-grado, las instituciones de educación superior algo pueden decir en ello, toda vez que quienes estudian educación parvularia lo hacen en ellas y, para todos y todas aquellos/as profesionales que sean profesores de danza y que se incorporen a la educación formal en instituciones educativas públicas o privadas, deberán enfrentar el tema de la formación de audiencia y aquellos que sean periodistas o similares y que se dediquen a la crítica en danza, tendrán que hacerse cargo de ello, ¿será que las instituciones de 
educación superior no tienen nada que hacer en ello? Otro espacio pleno de posibilidades de desarrollo y aporte.

En lo que concierne al lineamiento Patrimonio cultural se establece que ello es un problema a causa de la "Ausencia de mecanismos y espacios para preservar, rescatar y difundir el patrimonio de la danza." (C.N.C.A., 2010,p 48). Para éste se declaran al menos dos problemas específicos que constituyen otro gran espacio de participación: "Carencia de un espacio adecuado para el registro, conservación y preservación del patrimonio de la danza." y, "Escasez de investigaciones y reflexiones teóricas que permitan profundizar y promover la memoria y el desarrollo actual de la danza" (C.N.C.A., 2010,p 48). Resulta evidente que toda institución de educación superior cuenta como parte de su gestión la investigación y reflexión, hacerlo en danza es simplemente especificar el tema, por tanto, la generación de seminarios, espacios de encuentro docente para quienes ejercen la docencia en las carreras de danza, no debieran estar en cuestión, la pregunta es ¿dónde hay que inscribirse para participar de tal o cual actividad de la universidad $x$ ? $y$, en lo que concierne al registro y conservación de patrimonio, las instituciones de educación superior cuentan con bibliotecas, las que hoy son centros de recursos de variadas posibilidades y formatos. Cuando cada profesor piensa en los alumnos y alumnas a los cuales ha guiado en su proceso de titulación y revisa en formato físico o virtual el documento que da cuenta del trabajo de tesis realizado, sucede que en danza ello no alcanza, se requiere un registro visual, con buena imagen y audio apropiado, para ver en qué consistió ese trabajo de titulación más allá del documento que lo describe, no hay otra manera de llegar a un "arte escénica", su definición es ser en el momento de su presentación, pero pocas veces existe un registro, no constituyen un valor y una responsabilidad de la institución de educación superior, tratando un arte escénica como cualquier otra área del conocimiento, extraño, por decir lo menos.

Pero en lo que concierne al registro del patrimonio, puede pensarse no solo en lo que viene, sino también en la recuperación, todos esos espectáculos basados en la historia nacional, podrían ser un gran proyecto de instituciones de educación superior, pensar, por ejemplo, en el registro profesional que hoy no existe, pero debiera, del montaje que hiciera el ballet Pucará décadas atrás respecto de la Cantata Santa María, o el registro del montaje de las Mujeres del Carbón que hiciera hace poco el bailarín chileno de flamenco Pedro Fernández, son recursos que cualquier profesor de danza en la educación superior necesita para mostrarle a sus alumnos y alumnas, pero hasta el momento no hay cómo, generaciones de intérpretes, coreógrafos, directores en el mundo de lo efímero, la danza desaparece después de cada función, es partir de cero cada día cuando no hay registro. . 
En lo que concierne a cultura y artes, y danza comprendida en este marco, se cuenta con una gran ventaja: la institucionalidad en esta área es completamente actual, por tanto, el concepto de política pública es la base desde la que se construye la acción, de manera formal y declarada, lo que no resulta habitual en las demás áreas de intervención del Estado, donde existen los proyectos que ejecutan la política, la que se puede comprender, conocer y/o deducir desde los programas de gobierno de la administración en curso, lo que se conjuga con la misión y visión declarada por la respectiva cartera, pero un documento que declare de manera detallada la política pública de una repartición estatal, sobre la cual se constituya su gestión, hasta ahora solo sucede en cultura. No es un ciclo completo, faltan los estudios de resultado, los diseños de implementación, pero sucederá si los actores involucrados participan como deben.

No será fácil diseñar en conjunto, pues somos un país que parte de cero cuando el cambio de administración en el gobierno implica un cambio valórico, es como si la política pública no tuviera historia, se desestima el análisis sobre el cual se estableció el problema y la manera cómo resolver, se pierde el diseño, se recortan los recursos destinados, se desacredita la ejecución, se regresa no al ayer, sino al antes de ayer, pero en danza no hay cómo escabullirse cuando se trata de lo que podrían aportar las instituciones de educación superior, estamos hablando de lo que corresponde a la sociedad civil y al mundo privado en la danza de la política pública, es cuestión de aplicar el estilo cueca urbana, desafiante pero fluida, conquista mutua, con forma de cueca, con contenido de cueca para una vida donde bailar es parte del desarrollo social y una vida mejor.

\section{Referencias}

Consejo Nacional de la Cultura y las Artes. (2016). Política de fomento de la danza 20102015. Recuperado de https://www.cultura.gob.cl/wpcontent/uploads/2011/11/politica_danza.pdf

Hernández, R. Fernández, C. \& Baptista, P. (1997). Metodología de la investigación. Colombia: McGraw Hill Interamericana de México.

Lahera, P., (2002). Introducción a las políticas públicas. Santiago de Chile: Fondo de Cultura Económica.

Lasswell, H. (1963). Future of political science. Estados Unidos: Greenwood Press. 
Helena Hidalgo González

Licenciada en lengua y literatura hispánica, U. de Chile; Magíster Gobierno y Sociedad, U. Alberto Hurtado; Diplomada en: Ingeniería de la capacitación; Programa de Calidad y Equidad en la Formación; Agent de développement local. Profesional con especialización y experiencia en: Diseño, coordinación, sistematización y seguimiento de programas de Políticas Públicas; Docencia en enseñanza superior en lenguaje y literatura, arte y administración pública; Edición de libros, diseño de material instruccional y diseño curricular. Email: helena.hidalgo@gmail.com 